\title{
PIN POWER CALCULATION SCHEME FOR REACTOR PRESSURE VESSEL FAST NEUTRON FLUENCE ESTIMATION
}

\author{
Mariya Brovchenko ${ }^{1}$ and Julien Taforeau ${ }^{1}$ \\ ${ }^{1}$ Institut de Radioprotection et de Sûreté Nucléaire (IRSN) \\ 31 avenue de la Division Leclerc, 92620 Fontenay-aux-Roses, France \\ mariya.brovchenko@irsn.fr, julien.taforeau@irsn.fr
}

\begin{abstract}
The estimation of the neutron fluence at the Reactor Pressure Vessel (RPV) is classically carried out by a two-step approach. The first step is to estimate the full core neutron source term whether the second step of the calculation consists in the transport of neutrons from the core (source term) to the RPV using the neutron fission distribution determined in the previous step. For this purpose, the neutron fission distribution is to be accurately determined at the fuel pin level for the assemblies on the border of the core. To achieve this goal, two methods are evaluated in this study. The first method considered is a full core 2D Monte Carlo calculation using the MNCP6 code. The second method is based on a deterministic approach using the CASMO5 multi-segment option, allowing a full 2D transport calculation at the pin level with an expected accuracy similar to a stochastic method. The comparison of the two methods shows an overall good agreement with differences within the statistical uncertainty for different cores: homogeneous UOX core, mixed UOX-MOX loading and the effect of the hafnium rods used in the assemblies in the periphery of the core. The modelling limitation and the associated calculational time are discussed for the comparison of the two approaches.
\end{abstract}

KEYWORDS: core power distribution, pin-by-pin analysis, deterministic methods, Monte Carlo methods

\section{INTRODUCTION}

The lifetime of a nuclear reactor is above others related to the ageing of the reactor pressure vessel under neutron irradiation. Indeed, the embrittlement of the vessel material is primarily induced by the bombardment of fast neutrons. The estimation of the neutron fluence at the reactor pressure vessel (RPV) is classically carried out by a two-step approach [1]. The first step is to estimate the full core neutron source term, either Monte Carlo [1] or deterministic calculation [2] [3] or hybrid methods [4] can be used. The second step of the calculation consists in the transport of neutrons from the core to the RPV using the neutron fission distribution determined in the previous step, a standard procedure is to combine a stochastic calculation to evaluate the neutron attenuation with a variance reduction technique (weight window etc.). The quantification of bias and uncertainties in the estimation of the neutron term source is of first importance and can be due to nuclear data [5], geometrical uncertainties or approximation in the modelling [6] [7]. This work focuses on the first step of the calculation: the estimation of the neutron fission source term. Because the fluence at the vessel is strongly related to the fissions occurring at the periphery of the core, mainly the last two rows of assemblies contribute to the neutron fluence at RPV. More precisely, inside these two rows of assemblies, the fuel pins near to the core periphery are of higher importance than the pins closer to the center of the core. Consequently, the neutron fission distribution is to be accurately determined 
at the fuel pin level. To achieve this goal, two methods are evaluated in this study. The first method considered is a full core 2D Monte Carlo calculation using the MNCP6 code [8]. The second method is based on a deterministic approach using the CASMO5 [9] multi-segment option, allowing a full transport calculation at the pin level with an expected accuracy similar to a stochastic method.

This paper focuses on a systematic comparison of the two methods to evaluate the pros and cons of each method, starting from a very simple core management up to a real core configuration. The weaknesses in the Monte Carlo approach are linked to the hypotheses taken for the depletion calculation of the fuel composition and the thermal spatial dependence. This paper will address different cores without using the depletion part, which will be addressed in a next study.

Furthermore, regarding the deterministic approach, the accuracy of the fission distribution at the core/periphery will be addressed. In addition, impact of the multi-group treatment approach will be evaluated in this study.

\section{MODELLING AND METHODS}

The reactor considered is a French type of the Pressurized Water Reactor (PWR) 900MWe with UOX and UOX/MOX core. Several loadings are considered, a pure academic fresh fuel homogeneous UOX fuel core and UOX/MOX fuel loading. Some additional comparisons will be presented to verify the simulation of the hafnium rods inserted in the assemblies at the periphery of the core [10].

The core is surrounded by the baffle, the envelope and the radiation shielding that is not symmetric as shown in Figure 1 on the MCNP model. CASMO5 does not support an asymmetric radiation shield as shown in Figure 1. Several azimuths are of interest for the fluence calculations, leading to high importance of the fissions occurring in all the assemblies at the periphery of the core.
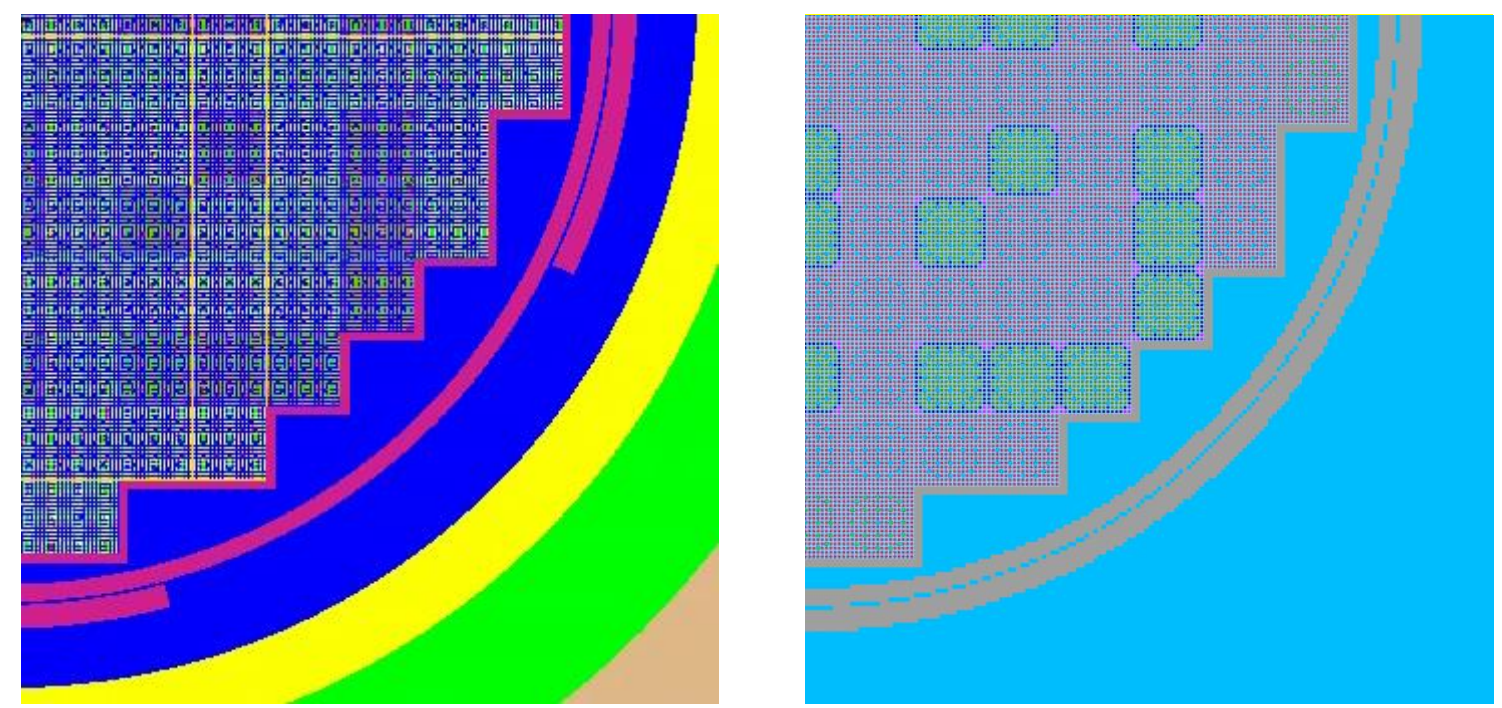

Figure 1. Reactor geometry modeling: MCNP (left) and CASMO5 (right).

Regarding UOX and MOX assemblies (Cf. Figure 2), a classical design has been considered. Each assembly contains a total of 264 pins including 24 empty guide tubes for control rods insertion and a central instrumented position. In the MOX case, a three zones pattern has been adopted: pin rods with a high enrichment in the central part and lower enrichments at the periphery and corners of assemblies. 

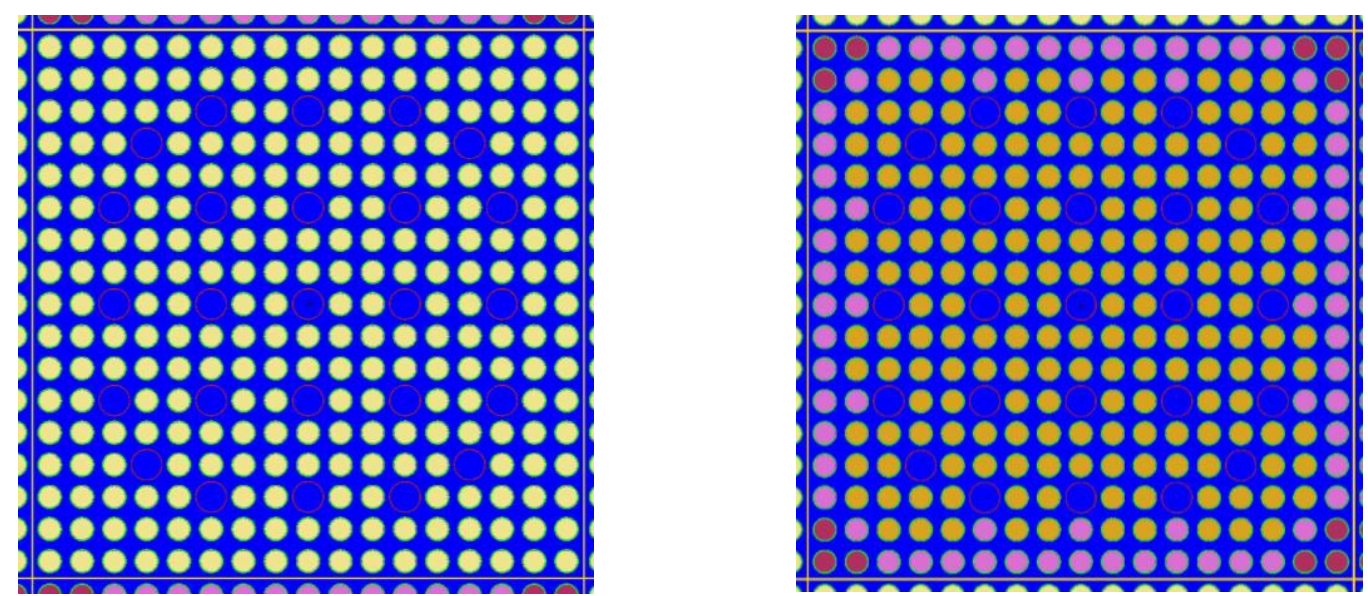

Figure 2. UOX assembly (Left); MOX assembly (Right) with 3 different types of fuel pins: high (orange), medium (rose) and low (red) Pu content.

The UOX/MOX core loading, presented in Figure 3, is a hybrid UOX/MOX core management. The choice of using a hybrid core loading is driven by the need to identify specificities in the MOX modeling (number of neutrons emitted by fission, fission spectrum, gradient of flux at the UOX/MOX interface ...).

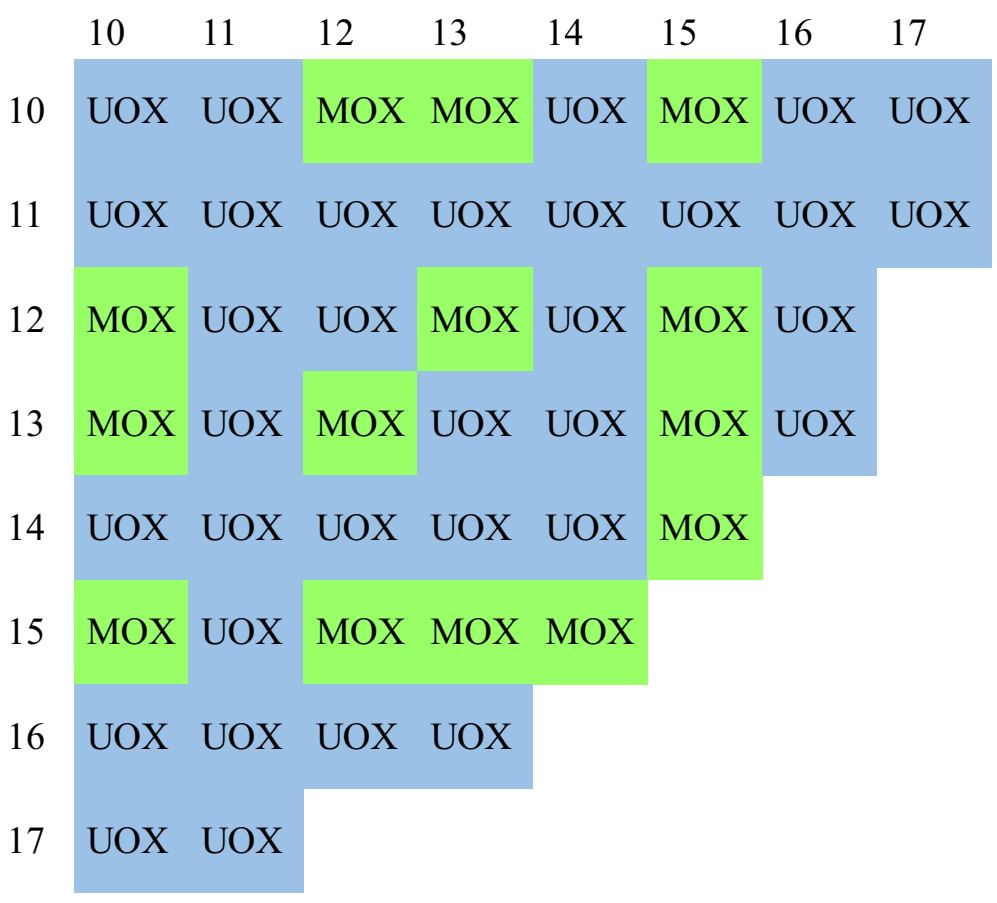

Figure 3. Mixed UOX-MOX core loading.

\subsection{MCNP6 core calculations}

In the MCNP model, the geometry of core, baffle, envelop, radiation shielding and the vessel can be modeled as precisely as wanted. In order to avoid the bias linked to the specific modelling of the radial reflector with CASMO, the thickness of the baffle was reduced to the value of $2.5 \mathrm{~cm}$. The radiation shield was kept asymmetric contrary to CASMO5 since no big effect of this hypothesis was expected and could 
not be observed. The geometry considered was limited to 1 meter height limited by surfaces with total reflection conditions above and below the core. The grids located in the core were not modeled for this comparison. No thermal hydraulic effects are considered in the model, the dimensions correspond to the temperature of $20^{\circ} \mathrm{C}$ for all the calculations. The gap between the fuel pin and the clad is modeled.

In order to have a correct estimation of the standard deviation, 50 to 120 different calculations were performed using the same starting fission distribution, but with different starting random seeds. The provided starting source was obtained for a similar core using 100000 particles per cycle. The number of cycles of the starting source was not counted precisely, but was large enough. The calculations were performed using 50000 particles per cycle and 500 active cycles after 300 inactive cycles. Thanks to the inactive cycles, the calculations are considered as independent results. The associated standard deviation is calculated based on the scores of the independent calculations without taking into account the standard deviations associated to each calculation estimated by MCNP, which was of the same order around 1-5\%. The simulation conditions may vary for different cores. Especially, for the cores with strong power decrease on the border of the core, additional calculations were added to obtain a sufficiently low standard deviation on the fuel pins with low power. The targeted standard deviation for the cores at the pin level of all presented calculations was $2 \%$. The computational effort associated to each studied core varies from around 200000 to $400000 \mathrm{~min}$ CPU in total.

The tallies used for the comparison were the fission rates and the number of neutrons emitted per fission multiplied by the fission rates. These scores were collected over the cells of the pins resulting in two times 41448 scores. Python scripts were used for the extraction and analysis of the scores. Due to the asymmetric radiation shielding, each fuel pin is treated separately. The core calculations were performed using ENDF/B-VII. 1 nuclear data library for all isotopes, except oxygen for which JENDL-3.3 nuclear data library was used. Indeed, in deep penetration problems MCNP encountered sometimes errors using ENDF/B-VII.1 nuclear data library for the ${ }^{16} \mathrm{O}$.

\subsection{CASMO5 core calculations}

The methodology used to model the core in the CASMO5 code is based on the multi-segment option (MxN). The calculation is performed in two steps: each assembly type is depleted separately, in an infinite lattice, to produce a restart file that is then used for the full core calculation.

The single assembly simulation is performed using the classical two-step calculation scheme (Pij (586 groups)/MOC(19 groups)). This kind of calculation scheme is used in lattice code such as APOLLO2 to provide homogenized cross sections at the assembly level for PWR modeling [11]. In order to be as close as possible to the Monte Carlo calculation, the following options are deactivated: thermal expansion, resonance upscatter model, effective Doppler temperature model and no spacer grids smeared in the coolant. The ENDF/B-VII.1 nuclear data library is considered for all major isotopes. Regarding the special case of assemblies containing Hafnium pins, the depletion is performed with hafnium inserted and then removed at a desired burnup step.

The 2D full core calculations (MOC) are performed at 35 energy groups, the baffle thickness is equivalent to 2 pins $(2.5 \mathrm{~cm})$. For each calculation, the pin by pin power (or fission rates) distribution is evaluated. However in this paper the results are limited to fresh cores, consequently, only direct full core calculation are needed. The calculation time is around $180 \mathrm{~min}$ CPU in total for each simulation of the core.

\section{RESULTS}

\subsection{UOX fresh fuel core}

The first comparison was performed on a very simple core using identical UOX assemblies with $3.7 \%$ enrichment in uranium 235 in the whole core. The fuel temperature was set to $900 \mathrm{~K}$, all other materials 
were at $578.15 \mathrm{~K}$. Concentration of boron was set to 0 . No thermal adjustment was used in CASMO. Fifty independent calculations were performed with MCNP, resulting in $180000 \mathrm{~min}$ CPU.

The $k_{\text {eff }}$ calculated by the two codes are provided in the Table 1 . The difference between the two evaluations is a bit higher than expected, but the reason for the higher deviation for this case compared to the following cases could not be found during this work, and will be further analyzed in the future.

Table I. Multiplication coefficient for UOX core: MCNP and CASMO results.

\begin{tabular}{|c|c|c|}
\hline & MCNP & CASMO \\
\hline $\mathrm{k}_{\mathrm{eff}}$ & $1.36760+/-29 \mathrm{pcm}$ & 1.36540 \\
\hline
\end{tabular}

The fission rate distribution was compared at the pin and the assembly level, see Figure 4. The assembly level comparison shows no significant impact. The random distribution of the discrepancies indicates its origin due to the statistical uncertainty in the Monte-Carlo calculation. The pin level comparison points out a very good agreement between the two codes, with discrepancies mainly within one standard deviation of $2 \%$, and some pins above this value. Even though the Monte-Carlo calculation is considered as the reference for the comparison, in this first case, the statistical uncertainty can be observed through the under-estimation on the fuel pins positioned at the periphery of the core and more precisely in the corner of assemblies. The errors are in the range from $-3.4 \%$ up to $6 \%$ demonstrating an overall good agreement in almost all locations.
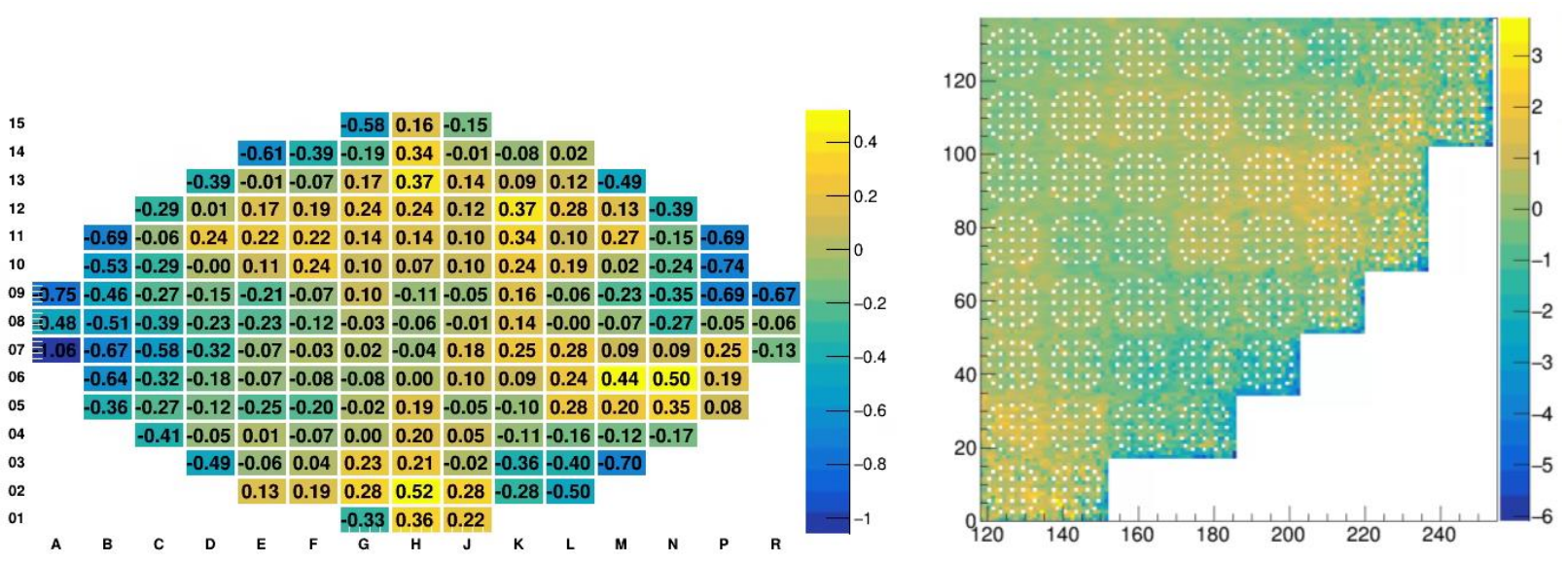

Figure 4. Fission rate distribution comparison (MCNP-CASMO/CASMO) on the assembly level and pin level.

\subsection{UOX/MOX}

In this case, a mixed UOX/MOX assemblies loading was simulated as shown in Figure 3. Fifty independent calculations were performed with MCNP to get the desired standard deviation on the pin fission rate, leading to a total of $150000 \mathrm{CPU}$ min. The $\mathrm{k}_{\text {eff }}$ results are provided in Table II and show a good agreement between the two codes. The differences of the fission rate distribution are presented in the Figure 5 left. The associated Root Mean Square (RMS) is of $1.04 \%$. The minimum and maximum errors are around 6\% located at the corner of border assemblies, however it has to be noticed that only $1 \%$ of the total pins are above 3\% demonstrating a very good agreement between CASMO5 and MCNP calculations. 
Table II. Multiplication coefficient for UOX/MOX core: MCNP and CASMO results.

\begin{tabular}{|c|c|c|}
\hline & MCNP & CASMO \\
\hline $\mathrm{k}_{\mathrm{eff}}$ & $1.28829+/-19 \mathrm{pcm}$ & 1.28772 \\
\hline
\end{tabular}
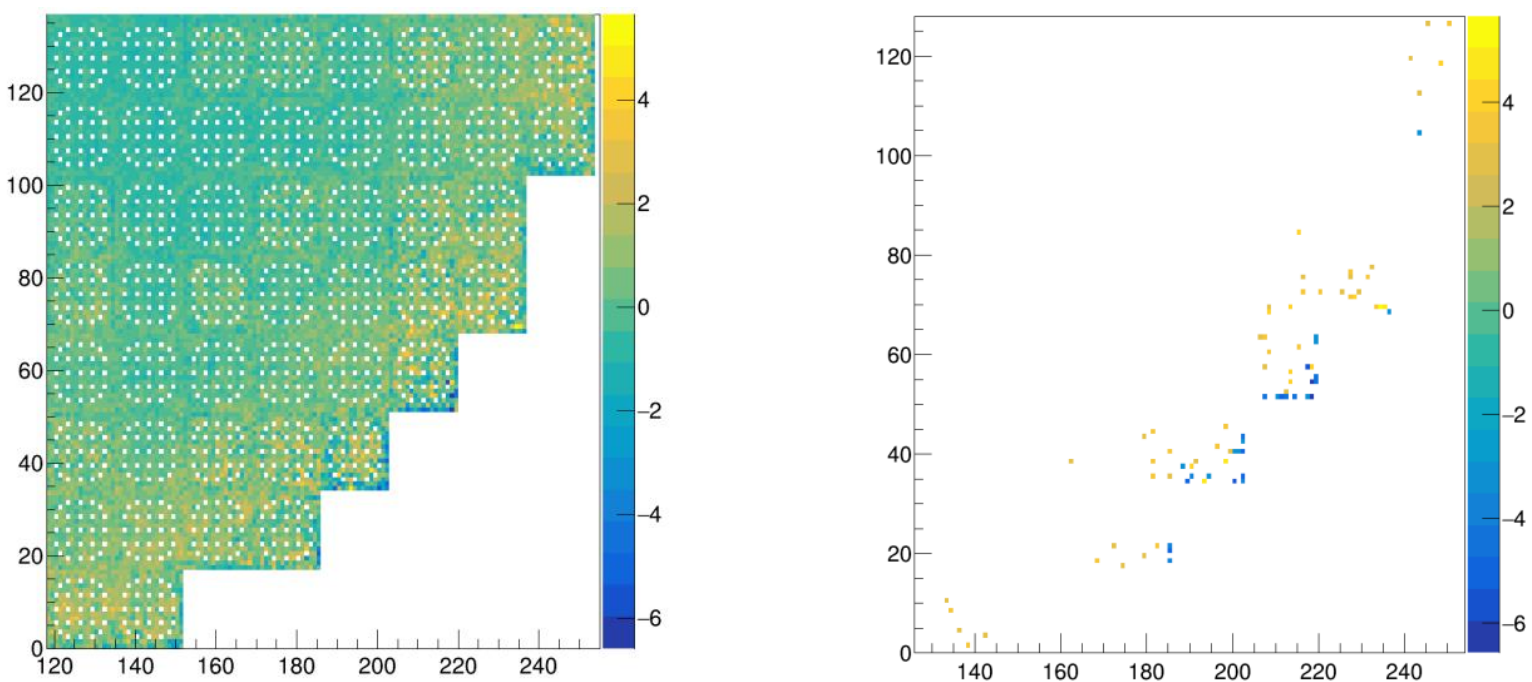

Figure 5. Fission rate distribution comparison (MCNP-CASMO/CASMO) on the pin level and filtering the discrepancies above $3 \%$ for a hybrid UOX/MOX core loading.

\subsection{UOX/MOX with Hf absorber rods fresh fuel core}

In this case, the same UOX/MOX assemblies loading was used as previously, while the three assemblies at positions $\mathrm{G}, \mathrm{H}$ and $\mathrm{J}$ (and symmetrics) at the periphery of the core contain Hf rods instead of the guide tubes in Figure 2. The instrumentation tube is unchanged filled with water.

120 independent calculations were performed with MCNP to get the desired standard deviation on the pin fission rate, leading to a total of $400000 \mathrm{CPUmin}$. The $\mathrm{k}_{\text {eff }}$ comparison shown in Table III indicates a good agreement between the two codes (less than $20 \mathrm{pcm}$ ).

Table III. Multiplication coefficient for UOX/MOX core with Hf rods: MCNP and CASMO results.

\begin{tabular}{|c|c|c|}
\hline & MCNP & CASMO \\
\hline $\mathrm{k}_{\mathrm{eff}}$ & $1.28723+/-29 \mathrm{pcm}$ & 1.28702 \\
\hline
\end{tabular}

The differences of the fission rate distribution are presented in the Figure 6 left. The associated RMS is of $0.76 \%$, even if for some fuel pins a discrepancy up to $4 \%$ can be noticed. As shown in Figure 6 right, only $3 \%$ of the pins leads to discrepancies above $2 \%$. The highest discrepancies are located in the assemblies containing the Hf rods, where the pin power is very low leading to statistical challenges for the MonteCarlo calculations. For this reason, the number of calculations had to be increased.

It should be noticed that, for UOX-MOX core loadings as well as for assemblies with different burn-ups, the fission rate and the neutron emission, as defined below, distributions should be distinguished.

$$
\text { Neutron emission }(\vec{x})=\text { number of neutrons emitted per fission }(\vec{x}) * \text { fission rate }(\vec{x})
$$


Neutron emission distribution is the physical quantity that should be used for the second step fixed source calculation of neutron attenuation up to the vessel surface. For the UOX homogeneous core, the difference between the fission rate and the neutron emission distributions is negligible. Indeed, the number of neutrons emitted by plutonium-239 and by uranium-235 fissions differs and leads to an effect of $17 \%$ more probability of emission for the MOX assemblies compared to the UOX assemblies as can be seen in Figure 7.
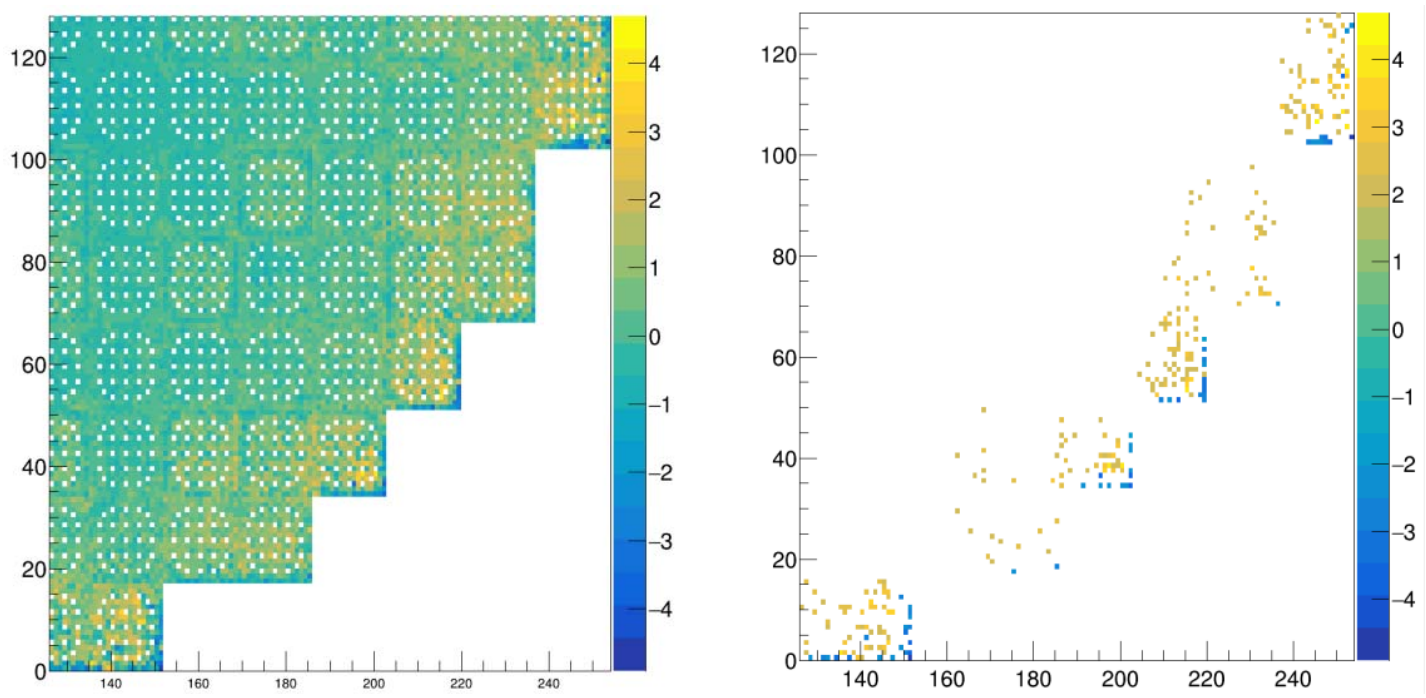

Figure 6. Fission rate distribution comparison (MCNP-CASMO/CASMO) on the pin level (left) for a hybrid UOX/MOX loading with Hf rods and filtering the discrepancies above $2 \%$ for a hybrid UOX/MOX core loading (right).

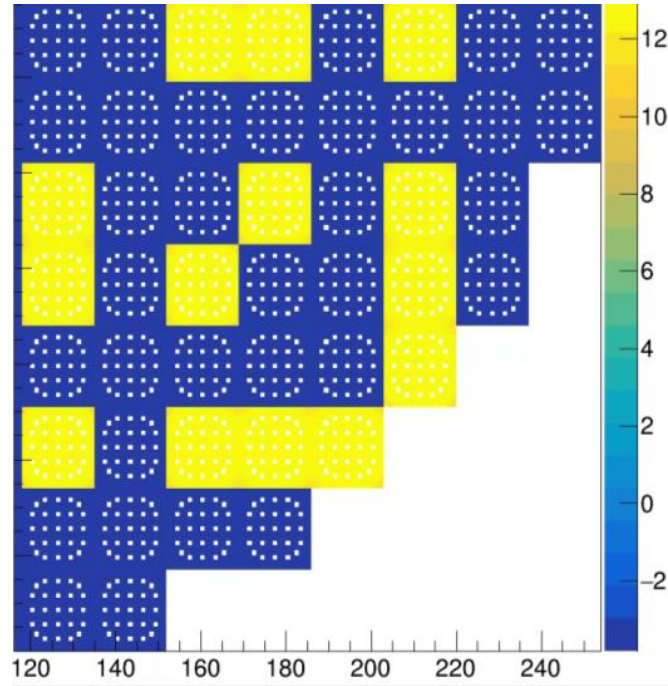

Figure 7. Neutron emission rate and fission rate distributions comparison ((neutrons fission)/neutrons) from MCNP. 


\section{CONCLUSIONS}

The problems linked to radiation transport outside the reactor core need a good description of fission neutrons emission in the core of the reactor. The major part of neutrons that hit the vessel and contribute to the fluence are emitted at the periphery of the core and more precisely in the last row of assemblies. This paper evaluates two procedures to estimate the neutron source term at a pin level based on Monte Carlo (MCNP) or deterministic transport method (CASMO). From this study, it appears that both methods are in good agreement and give consistent results with less than $6 \%$ of difference in all cases studied. Three points should be underlined: the calculation time which is thousand times faster for deterministic approaches to obtain a similar accuracy, then the fact that the major differences between the two methods are found at the last pin row of border assemblies, if this point is not problematic to evaluate the general core behavior, it has to be considered in the frame of the evaluation of fast fluence at the reactor vessel. Finally, in the case of hybrid core management, the fission rate and the neutron emission distributions should be distinguished leading to significant differences in the source term evaluation (up to $17 \%$ ). Similar work will be performed on cores with depletion effects. Indeed, the depletion is performed taking some additional hypothesis that will be quantified through the comparison of Monte-Carlo and deterministic approaches. As underlined in this paper, the difference between effects on the neutron emission and fission rate distributions will be pursued.

\section{REFERENCES}

1. M. Brovchenko, B. Dechenaux, K.W. Burn, P. Console Camprini, I. Duhamel, and A. Peron. "Neutrongamma flux and dose calculations in a Pressurized Water Reactor(PWR)." Proceedings of the ICRS-13, Paris, EPJ Web of Conferences, volume153, p. 05008 (2017).

2. C. Gosmain, C. Sandrin, M. Tommy-Martin, I. Rupp and J.L. Flejou, "Impact on Neutronic Calculation Of Thermomechanical Expansion of Reactor Vessel Internals", $23^{\text {rd }}$ Int. Conf. Nucl. Ene. For New Europe, Slovenia, (2014)

3. A. Vasiliev, H. Ferroukhi, M. A. Zimmermann, R. Chawla, "Development of a CASMO-4/SIMULATE3/MCNPX calculation scheme for PWR fast neutron fluence analysis and validation against RPV scraping test data", Annals of Nuclear Energy, Volume 34, Issue 8, 2007, Pages 615-627, https://doi.org/10.1016/j.anucene.2007.02.020.

4. P.G. Laky and N. Tsoulfanidis, "Neutron Fluence at the Pressure Vessel of a Pressurized Water Reactor Determined by the MCNP Code", Nuclear Science and Engineering, 121:3, 433-447, DOI: 10.13182/NSE95-A24145 (1995)

5. L. Clouvel , P. Mosca , J.M. Martinez, and G. Delipei "Shapley and Johnson values for sensitivity analysis of PWR power distribution in fast flux calculation", M\&C 2019, Portland, OR, August 25-29, 2019

6. R. Vuiart, M. Brovchenko, and J. Taforeau, "Impact of core power variations on the fast neutron flux incident on pressurized water reactor vessels", ICAPP 2019, Juan-les-pins, France, May 12-15

7. M. Brovchenko, I. Duhamel, B. Dechenaux, "Neutron-gamma flux and dose calculations for feasibility study of DISCOMS instrumentation in case of severe accident in a GEN 3 reactor", Proceedings of ICRS-13 \& RPSD-2016, October 3-6, Paris, France.

8. T. Goorley et al., "Initial MCNP6 Release Overview - MCNP6 version 1.0", LA-UR13-22934, (2013)

9. J. Rhodes \& al., "CASMO5 A fuel Assembly Burnup Program User's Manual", Rev8, Studsvik Scandpower Inc (2014)

10. Westinghouse, "Peripheral Power Suppression Assembly", September 2015 www. westinghousenuclear.com/Portals/0/flysheets/NF-FE-0050 PPSA.pdf

11. J.F. Vidal \&al., "New modelling of LWR assemblies using the APOLLO2 code package", M\&C 2007, Monterey, CA, April 15-19, 2007 